\title{
Investigaciones y estrategias comunicativas frente a la violencia contra las mujeres en ámbitos universitarios. Una revisión ${ }^{8}$
}

\author{
Laura Cristina Bonilla Neira \\ Doctora (C) en Lingüística \\ Universidad de Buenos Aires \\ Argentina \\ Correo electrónico: laura.bonilla369@gmail.com
}

\section{Diana Carolina Gómez Meneses \\ Magister en Psicología Universidad Pontificia Bolivariana Bucaramanga, Colombia Correo electrónico: diana.gomezm@upb.edu.co}

\author{
Juan Carlos Godoy Pinilla \\ Magíster en gestión de la tecnología educativa \\ Universidad Pontificia Bolivariana \\ Bucaramanga, Colombia \\ Correo electrónico: juan.godoy@upb.edu.co
}

Recibido: $17 / 10 / 2017$

Evaluado: 25/06/2018

Aceptado: 13/07/2018

\section{Resumen}

El objetivo de este artículo es realizar una revisión sobre las investigaciones en materia de violencia de género y de las estrategias comunicativas que se han diseñado e implementado en las universidades nacionales e iberoamericanas para visibilizar y sensibilizar sobre esta violencia. Se utilizó una metodología cualitativa con un diseño hermenéutico y perspectiva de género para recolección y análisis. Se encontró un vasto trabajo de investigación sobre la violencia de género en España y Norteamérica y un inicio en Latinoamérica, así como algunas iniciativas en materia de comunicación. Se discute la poca articulación que tienen las investigaciones con las campañas contra la violencia de género dentro de las universidades, así como la falta de compromiso de las instituciones con este problema de salud pública que poco a poco se está haciendo visible con más fuerza en las universidades con la movilización estudiantil.

\section{Palabras clave}

Violencia de Género, Estudios de Género, universidad, estrategias comunicativas.

8 Para citar este artículo: Bonilla-Neira, L., Gómez-Meneses, C., \& Godoy-Pinilla, J.C. (2019). Investigaciones y estrategias comunicativas frente a la violencia contra las mujeres en ámbitos universitarios. Una revisión Informes Psicológicos, 19(1), pp. 141-162 http://dx.doi.org/10.18566/infpsic.v19n1a08 


\section{Research and communication strategies in the face of violence against women in university environments. A review}

Abstract

The aim of this article is to conduct a review of research on gender violence and communication strategies that have been designed and implemented in national and Latin American universities to raise awareness on this type of violence. A qualitative methodology with a hermeneutic design and gender perspective was used for data collection and analysis. A vast research work was found on gender violence in Spain and North America and some in Latin America, as well as some initiatives in the field of communication. The lack of articulation that research has with campaigns against gender violence within universities is discussed, as well as the lack of commitment of institutions to this public health problem that is gradually becoming more visible within the universities thanks to student mobilization.

Keywords

Gender violence, gender studies, university, communicative strategies.

\section{Pesquisas e estratégias comunicativas perante a violência contra as mulheres nos âmbitos universitários. Uma revisão Resumo}

0 objetivo deste artigo é realizar uma revisão sobre as pesquisas que tratam a temática da violência de género e das estratégias comunicativas que se têm delineado e implementado nas universidades nacionais e ibero-americanas para visibilizar e criar sensibilidade ao respeito desta violência. Implementou-se uma metodologia qualitativa com um delineamento hermenêutico e perspetiva de género para a coleta e analise. Achou-se um vasto trabalho de pesquisa ao respeito da violência de género na Espanha e na América do Norte e um começo na América latina, assim como algumas iniciativas em matéria de comunicação. Discute-se a pouca articulação que as pesquisas têm com as campanhas em contra da violência de género ao interior das universidades, assim como a ausência de comprometimento das instituições com esta problemática de saúde publica que aos poucos tem-se tornado visível com mais força nas universidades com a mobilização estudantil.

\section{Palavras chave}

Violência de gênero, estudos de gênero, universidade, estratégias de comunicação. 


\section{ntroducción}

En la actualidad, la violencia contra la mujer se considera una problemática de salud pública con una alta prevalencia a nivel mundial. La OMS (2014) reporta que, alrededor de una de cada tres mujeres en el mundo, ha sufrido violencia física o sexual, de pareja, o violencia sexual en algún momento de su vida. Diversas organizaciones (Amnistía Internacional, 2017; OIT, 2005; ONU, 2013) han declarado esta situación como un problema social, político y de salud que aqueja a la población mundial. En Colombia, de acuerdo con el informe Comportamiento de la Violencia de Pareja del Instituto Nacional de Medicina Legal y Ciencias Forenses (Acosta, 2015), las mujeres son la población más afectada por este tipo de violencia con un $86.66 \%$, sobre todo las jóvenes, dado que el $43 \%$ del total de las víctimas se concentró en personas entre los 20 y 29 años.

La alta prevalencia y generalización del problema de la violencia contra las mujeres en Colombia, específicamente por su condición de género femenino, ha encendido las alarmas en materia legislativa y a nivel institucional. El Gobierno nacional, a través del Ministerio de Protección Social y la Consejería para la Equidad de la Mujer (2015), desarrolló un conjunto de estrategias por medio del Observatorio de Asuntos de Género para atender oportuna y diferencialmente los impactos desproporcionados que tiene la violencia basada en el género sobre las mujeres. Algunos avances legislativos, como la Ley 1257 (2008) en la cual se dictan normas de sensibilización, prevención y sanción de formas de violencia y discriminación contra las mujeres, junto a la Ley 1542 (2012) la cual pretende garantizar la protección y diligencia de las autoridades en la investigación de delitos de violencia contra la mujer en el Código Penal colombiano y la Ley 1761 (2015), que penaliza el delito de Feminicidio en Colombia.

Es importante enfatizar que dichos avances en materia legislativa son promovidos también desde el ámbito internacional por organizaciones como Amnistía Internacional (2017) y la ONU (2016), las cuales tienen dentro de sus objetivos la prevención de la violencia basada en el género, pues se ha reconocido que ésta es un impedimento para el avance y desarrollo de las sociedades. Así lo afirman Romero-Sánchez, López, Carretero-Dios y Rincón (2012a), cuando advierten que tanto mujeres como hombres desconocen las manifestaciones de la violencia al estar insertos en una cultura cargada de creencias que impiden identificar sobre todo la violencia de carácter psicológico. De ahí la importancia de la comunicación efectiva, en la cual la publicidad cumple un papel fundamental al influir con una intención persuasiva a la audiencia (Fernández, 2008). Así, las estrategias comunicativas están en la base de los procesos de sensibilización de estas problemáticas.

Debido a que la publicidad cumple la función de "elemento de socialización, entendiendo este como la adquisición y conocimiento de normas y valores de conocimiento básicos y aceptados en un grupo de pertenencia" (Fernández, 2013, p. 413), se hace necesario el diseño e implementación de estrategias comunicativas que permitan, por un lado, evidenciar las manifestaciones de violencia 
más sutiles e implícitas y, por otro, empoderar a las mujeres y a la sociedad en general frente a cómo prevenir, atender y actuar frente a estas situaciones. De hecho, un estudio realizado en España refiere que la comunicación relacionada con la igualdad de género "persigue sensibilizar a la población y, por tanto, propone como objetivo un cambio en las opiniones y actitudes" (Papí \& Orbea, 2011, p. 249). Incluso, afirman que este tipo de publicidad persigue un cambio de conducta en la población.

Como se dijo, en Colombia, la Ley 1257 de 2008 señala la importancia de sensibilizar y empoderar para prevenir la violencia de género. En el Art. 9 de esta Ley se insta a las autoridades encargadas a formular e implementar políticas que reconozcan las diferencias y las desigualdades sociales, entre estas las de género. Asimismo, el Art. 11 se refiere a las medidas educativas, específicamente señala que el Ministerio de Educación (MEN) tendrá la función de "velar para que las instituciones educativas incorporen la formación en el respeto de los derechos, libertades, autonomía e igualdad entre hombres y mujeres" (2008, p.8). Además, en el apartado tercero del mismo artículo, se determina que el MEN debe desarrollar políticas que contribuyan a sensibilizar, capacitar y entrenar a la comunidad educativa, especialmente estudiantes y docentes frente a la violencia contra las mujeres, pues se debe abordar el tema de igualdad de género y no discriminación de forma transversal (Ley 1257, 2008). De manera que se reconoce a las instituciones educativas, entre éstas las universidades, como espacios para la formación en materia de género, así como de sensibilización de este tipo de violencia.

\section{Violencia de género en el ámbito universitario}

La violencia de género hace referencia a todos los actos que violan los derechos humanos de las mujeres y las niñas, establecidos en la Conferencia Mundial sobre los derechos humanos en 1993 (ONU, 1999), en la cual se explicitan por primera vez los derechos propios de las mujeres. En Colombia, la Ley 1257 de 2008 comprende la violencia hacia las mujeres como "cualquier acción u omisión, que le cause muerte, daño o sufrimiento físico, sexual, psicológico, económico o patrimonial por su condición de mujer, así como amenazas de actos, la coacción o la provocación arbitraria de la libertad" (2008, p.1). De acuerdo con la Declaración de la Cuarta Conferencia Mundial sobre la Mujer, celebrada en Beijing (ONU, 1999), las consecuencias de la violencia involucran afectaciones a nivel físico, psicológico, comportamental y sexual, entre otros. A nivel físico, se pueden sufrir lesiones, discapacidad o hasta la muerte. En cuanto a la salud mental, presentar trastornos depresivos, del sueño, alimentarios, estrés, ansiedad, baja autoestima, intentos o suicidio. En materia comportamental, el uso de drogas, las conductas de riesgo en la sexualidad o elección de parejas abusivas, y a nivel de la sexualidad, acontecer situaciones como el aborto, enfermedades de transmisión sexual, disfunción sexual, entre otras.

En el ámbito universitario, uno de los fenómenos que ha tenido especial atención ha sido el del acoso sexual, el cual ha sido abordado en investigaciones de Estados Unidos (Hayes-Smith, 2010; Hoxmeier et al., 2016) y España (Bosh-Fiol, 
2009; Romero-Sánchez et al., 2012b). Estos estudios han estado motivados por denuncias de estudiantes y, por supuesto, el interés de los y las investigadoras. Al respecto, Bosh-Fiol (2009) menciona que el acoso sexual, de acuerdo a lo explicitado por la Organización Internacional del Trabajo, se define como "aquel comportamiento de naturaleza sexual o de connotaciones sexistas, no deseado por la víctima, que afecta a la dignidad de mujeres y de hombres" (OIT, 2005, p.2) lo cual revela que la degradación de la mujer supone un acto de maltrato.

Asimismo, la violencia de género en las universidades se presenta como maltrato por superiores o colegas y puede ser recibido en entornos laborales o académicos, siendo éste señalado como acoso laboral. De hecho, estamentos como la Organización Internacional del Trabajo (OIT, 2005) reconoce el acoso como un problema de seguridad y salud y que, en caso particular del acoso sexual, constituye una manifestación de la discriminación basada en el género. Una de las causas identificadas por los investigadores obedece al establecimiento de una serie de mitos o creencias que se reproducen en el imaginario social y pretenden justificar la presencia de la acción violenta y sus consecuencias (Ferrer, Bosh-Fiol, Ramis, Torres \& Navarro, 2006). En este sentido, los agresores presentan numerosas creencias distorsionadas sobre los roles de género, la inferioridad de la mujer y, sobre todo, la legitimación de la violencia como forma de resolver situaciones de conflicto (Echeburúa, Amor, Sarasua, Zubizarreta \& Holgado-Tello, 2016).

Por otro lado, los estudios sobre violencia de género (Buquet, Cooper, Mingo \& Moreno, 2013; Valls, 2008) han permitido analizar este fenómeno como un proceso y, al mismo tiempo, un resultado que representa la desigualdad entre hombres y mujeres en los roles cumplidos dentro de la sociedad, sean estos en el ámbito público o privado. Además, estos roles se configuran como puentes que visibilizan una realidad no tan reconocida y difundida y, en algunos casos, hasta deslegitimada a causa de creencias o mitos establecidos en una comunidad (Bosh-Fiol \& Ferrer-Pérez, 2012), más aún en el ámbito universitario, donde en las dos últimas décadas se ha investigado esta situación.

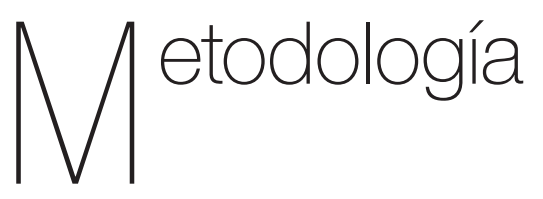

La metodología del presente trabajo tiene un enfoque cualitativo (Hernández, Fernández \& Baptista, 2010) por su flexibilidad y circularidad en la búsqueda de referencias válidas que permitan apropiarse de la mejor forma de las realidades sobre las investigaciones y estrategias comunicativas relativas a la violencia de género en el ámbito universitario. El diseño metodológico es hermenéutico (Berenguera et al., 2014) con perspectiva de género (Scott, 1996). Esta doble perspectiva permite acercarse a los significados e interpretaciones de los estudios encontrados sin dejar de lado el contexto histórico social y cultural al que pertenecen y así explorar las relaciones en las cuales se articula el poder desde una perspectiva amplia e inclusiva a la vez.

Para la ubicación de los documentos bibliográficos se recurrió a las bases de datos académicas multidisciplinarias disponibles como: EBSCO, Science Direct, Jstor y Google Académico. Se realizó la 
búsqueda bibliográfica entre noviembre de 2016 y febrero de 2017 utilizando los descriptores: violencia género universidades, violencia contra mujeres campus universitario, estrategias comunicativas, campañas publicitarias universitarias. Se tuvieron en cuenta artículos publicados entre 2007 y 2017 y algunos canónicos de los años 90 que han marcado la pauta en investigaciones de violencia de género en el ámbito universitario; así como tesis y comunicaciones de eventos internacionales.

Los criterios de selección se basaron en el tema de violencia de género en las universidades o con estudiantes universitarios; se hizo énfasis en investigaciones latinoamericanas con el objetivo de visibilizar más este entorno y se tuvieron en cuenta las diversas formas de violencia de género: sexual, física, psicológica y simbólica. A la selección de artículos publicados en revistas científicas se les sumó la recolección de textos publicados en la prensa colombiana con el objetivo de mostrar la visibilización de este tema y la posible articulación entre las investigaciones científicas realizadas en los claustros y la divulgación en los medios de comunicación más cercana a la sociedad en general.

\section{Norteamérica y España}

La problemática de la violencia de género afecta a las mujeres en todos los ámbitos sociales, sin distinción de estratos, edades o culturas. Así, la universidad como espacio social, también está permeada por estereotipos, prejuicios y creencias en torno a la violencia contra las mujeres. De hecho, las instituciones académicas prefieren mantener este flagelo en secreto y presumir la culpabilidad de las mujeres, dando la impresión de que el permitir una amistad con un hombre incluye por derecho actividades sexuales (Rubio, 2015). De ahí que, trabajos de visibilización, prevención y atención de la violencia de género se concentren en la idea de romper el silencio e invitar a la denuncia para hacer visible esta situación (Rozo, 2016; Valls, Puigvert, Melgar \& García-Yeste, 2016|). Los resultados de las recientes investigaciones en conjunto con el trabajo realizado por grupos activistas estudiantiles ante la discriminación y el acoso por razones de género han permitido acercarse a esta realidad, de modo que se han generado espacios de socialización y, en ese sentido, también de erradicación de esta violencia en el ámbito universitario.

Diversos estudios desarrollados sobre violencia de género en el ámbito universitario han revelado cifras alarmantes sobre el acoso y la discriminación que se vive al interior de estos centros educativos. En esa línea, Strauss (2004) señala que en Canadá y en Estados Unidos del 20\% al $40 \%$ de los estudiantes reportan una o más agresiones sexuales en el año en el que fueron encuestadas (Sugarman \& Hotaling, 1989, citados por Strauss, 2004). A su vez, otros investigadores han denominado a la universidad como "zona roja" para las jóvenes estudiantes (Flack et al., 2008; Mohler-Kuo, Dowdall, Koss \& Wechsler, 2004), porque se encuentran en riesgo creciente de experimentar contactos sexuales no deseados en el espacio universitario. De esta manera, las investigaciones han mostrado que los campus universitarios pueden constituirse en territorios hostiles para las mujeres. Osborne (1995) citado por Valls (2008) desarrolló 
una investigación en Canadá en la que mostró que la universidad resulta ser un contexto donde se desarrollan comportamientos y prácticas institucionales que están dirigidas a ejercer distintas formas de control social sobre las mujeres, a través de la fuerza, el abuso o el silencio.

La gran mayoría de los estudios sobre violencia de género en las universidades se han realizado en instituciones norteamericanas y es allí también donde se encuentra el mayor número de oficinas de atención, así como de medidas preventivas contra la violencia de género (Palaudi, 2016; Schewe et al., 2011). Por ejemplo, Fonow (1992) citado por Aguilar y otros (2009) revela que, de las violaciones ejecutadas en los campus universitarios en los EEUU, el 54\% suceden en las citas; también, que el 35\% de los jóvenes universitarios afirman cometerían una violación si tuvieran la oportunidad. En otro estudio, también citado por Aguilar (2009), se devela que un 95\% de las mujeres de una hermandad femenina universitaria quienes habían sufrido algún ataque sexual conocían a su agresor, es decir, hacía parte de su entorno cercano o incluso era su pareja.

Entre el 2004 y 2014, las investigaciones en violencia de género han tomado fuerza en las universidades españolas (Rodríguez, Lameiras, Carrera \& Alonso, 2014; Valls, Oliver, Sánchez, Ruiz \& Melgar, 2007), en algunos casos incluso financiado por el Instituto de la Mujer y el Ministerio de Trabajo y Asuntos sociales (2006-2008). De estos primeros estudios en España (Oliver \& Valls, 2004) se han desprendido otros trabajos que permiten esclarecer aún más la problemática $y$, en esa medida, han logrado implementar medidas para la superación de la violencia contra las mujeres en el ámbito universitario (Aguilar et al., 2009; Valls et al., 2014).

\section{Latinoamérica}

También en Latinoamérica se han realizado estudios que han posicionado la violencia de género en las universidades como un tema de gran relevancia para los investigadores, aunque no del mismo modo para las instituciones. Las investigaciones desarrolladas en torno a este tema en América Latina, como en las demás latitudes, han estado enmarcadas por hechos violentos sucedidos en los campus, situaciones que han impulsado las manifestaciones estudiantiles (Barrancos, Torricela \& Vázquez, 2015; Rozo, 2016) y del profesorado, quienes, de forma soterrada, han hecho visibles situaciones de discriminación, acoso y violencia sexual sufridas en su gran mayoría por las mujeres. Tal es el ejemplo presentado por Buquet y otros (2013) en su libro "Intrusas en la Universidad", el cual retoma investigaciones realizadas en otras latitudes que demuestran el ambiente hostil generado para las mujeres en el ámbito académico, evidenciando una cultura de rechazo hacia ellas a través de un ambiente de frialdad producto de conductas discriminatorias:
Un "clima frío" en el salón de clases es indicativo de "discriminación" en la medida en que pone en desventaja a niñas y mujeres, estudiantes de color, gays, lesbianas, bisexuales y transgénero, integrantes de las clases populares y personas con discapacidades (p. 49).

En México, también se han realizado estudios sobre actitudes y conductas 
asociadas a la sexualidad en estudiantes universitarios (Moral \& Ortega, 2008) y sobre la percepción de la violencia de género en jóvenes universitarios que hacen parte de estudios sobre juventudes y violencia en las universidades. Chávez y Rodríguez (2015) indagaron a través de tres instrumentos, uno cuantitativo y dos cualitativos, sobre el concepto de violencia de género, la aceptación y valoración de ésta, así como la presencia de sexismo benevolente. Entre los hallazgos se encuentra la alta prevalencia en la idea que las mujeres necesitan ser protegidas $y$, frente a la violencia, son la física y la sexual las que obtienen mayor prevalencia respecto a la simbólica.

En otro estudio, Chávez (2015) encontró que los jóvenes estudiantes de Trabajo Social de la UNAM presentan vacíos de información y conocimiento sobre los comportamientos que evidencian desigualdad de género, lo cual les impide reconocer la igualdad de género y, por esa vía, aceptan los roles de género impuestos socialmente. De hecho, una investigación realizada en la Universidad de Chapingo (Castro \& Vázquez, 2008) reafirma la idea que los roles de género se configuran desde la infancia, desde la perspectiva de la dominación masculina de Bourdieu (2000) y se reproducen en un ambiente adverso para las mujeres como la universidad, donde se puede vivir en un silencio social por temor al desprestigio institucional (Bernal, 2016).

A la par de los estudios llevados a cabo en diversas universidades en México sobre la violencia de género (Barbosa, 2015; Buquet, 2011), se ha aumentado también el apoyo de instituciones de investigación como el Conacyt (Ordorika, 2015), que, en conjunto, han coadyuvado a generar avances de carácter normativo y estructural en varias de ellas con la creación de políticas de género. Por ejemplo, la UNAM, que cuenta con el Centro de Investigaciones y Estudios de Género (ClEG), contribuyó en la reforma al Estatuto General de la Universidad, en la que se incluye un apartado sobre la igualdad entre hombres y mujeres; esto fue el impulso para la Comisión Especial de Equidad de Género (Buquet et al., 2013).

En este sentido, en Argentina se han realizado estudios sobre la violencia en el noviazgo en jóvenes universitarios como el de Miracco y Lanzilloti (2016), en el cual se indagan por las conductas con el vínculo entre dos personas que no conviven y cómo sobre estas parejas inciden diversas normas y creencias socio-culturales que sustentan conductas agresivas que, según las autoras, podrían sostenerse en el tiempo y reforzarse en la convivencia. Estas conductas llevarían a abordar más adelante la violencia de género en el ámbito intrafamiliar. Además, se explora en este estudio la presencia de conductas de control a través de dispositivos electrónicos mediante internet. La discusión gira en torno a la importancia de analizar la violencia en los jóvenes universitarios para favorecer la prevención y atención temprana del maltrato. Asimismo, se encontraron estudios específicos que miden la violencia de género en las universidades. Un estudio de la Universidad de Córdoba, Argentina, refiere que la violencia de género pasa no solo por los actos físicos sino también por las diferencias que atraviesan los estudiantes en su vivencia de ser hombres o mujeres (Domínguez, Fernández y Fuentes, 2013); por ejemplo, cursar carreras feminizadas o masculinizadas es enfrentarse a territorios complejos. Los estudiosos manifiestan que los 
territorios más hostiles para las mujeres son aquellos donde hay mayor cantidad de varones (aulas, canchas, incluso pasillos) lo cual puede conducir a que las mujeres enfrenten situaciones incómodas que dificultan su paso por la universidad. Sin embargo, los autores aclaran que, al mismo tiempo, las mujeres ponen en funcionamiento una serie de estrategias para enfrentar los obstáculos.

Asimismo, Vázquez, Palumbo y Fernández (2016) han abordado esta problemática haciendo una reflexión inicial sobre los límites e incumbencias de las instituciones de educación superior en cuanto al tratamiento de los casos. Las investigadoras han determinado que "si bien la Universidad no ejerce funciones supletorias de la justicia civil y/o penal, posee facultades disciplinarias que le permiten tomar medidas respecto de aquellas conductas que acontezcan o impacten en su ámbito" (Vázquez et al., 2016, p.110) y que, además, sean contrarias a los reglamentos vigentes en las universidades, y, en ese sentido, se ha avanzado más en la forma de afrontar los casos de violencia.

Además, en Argentina, después de un arduo camino desde la vuelta de la democracia (Barrancos et al., 2015) y a partir de situaciones de inflexión (como lo fue el caso del doble feminicidio de una estudiante universitaria argentina y su madre), se ha establecido la Red Interuniversitaria contra la violencia de género, en la cual se han juntado esfuerzos de todas la universidades adscritas con la intención de generar estrategias para la prevención, atención y sanción de estos casos en claustros universitarios (Barrancos et al., 2015). Esta red ha permitido un trabajo colaborativo que pretende que todas las universidades cuenten con mecanismos para enfrentar de forma efectiva la violencia de género.

En Costa Rica, se halló un estudio sobre las reacciones y efectos del hostigamiento sexual en población estudiantil (Carvajal \& Delvó, 2010), en el cual, a través de una investigación cuantitativa, se midió la prevalencia de este fenómeno en las distintas sedes de la Universidad Nacional costarricense. Algunos de los resultados arrojaron que una de cada tres estudiantes ha sido objeto de, al menos, una de las manifestaciones de hostigamiento sexual. Además, el $86 \%$ de los y las estudiantes que indicaron hostigamiento manifestaron que no lo comentaron ni denunciaron, lo que, a juicio de los investigadores, favorece la impunidad para lo cual se sugiere mantener y fortalecer los programas institucionales de prevención para la conciencia de la comunidad universitaria sobre la violencia de género.

En Ecuador, particularmente en Quito, se presenta una investigación sobre el imaginario de los estudiantes universitarios sobre el concepto de violencia de género (Barredo, Cevallos \& Berenguer, 2014). Dentro de los hallazgos de la investigación se presenta un tradicionalismo cultural patriarcal, dado que "se ha percibido en datos que casi cinco de cada diez estudiantes quiteños admiten que la manera de vestir puede incitar a la violación" (Barredo et al., 2014, p. 528). A pesar de ello, los investigadores afirman que ha habido un avance en las políticas, aunque la publicidad presenta un atraso. Esto evidencia, según el estudio citado, una prevalencia de actitudes conservadoras basadas en la desinformación, así como el poco impacto que presentan las 
campañas gubernamentales frente a la masiva publicidad sexista de las marcas.

En Venezuela, se encontró una tesis sobre violencia en el noviazgo desde la perspectiva de género en estudiantes universitarios. El documento (Lorenzo \& Salazar, 2011) toma una muestra de estudiantes con una edad promedio de 23 años en dos universidades en Caracas de varios programas académicos. Se destaca que un $21 \%$ de las estudiantes participantes declaran haber conocido a su pareja en la universidad. De acuerdo a los resultados, se evidenció un porcentaje de violencia física y sexual bajo; sin embargo, en la violencia psicológica los niveles se elevan, lo que, según las investigadoras, es muestra de que "la violencia es un proceso evolutivo y acumulativo que se manifiesta e intensifica con el pasar del tiempo y que su expresión inicial es, por lo general, violencia psicológica" (Lorenzo \& Salazar, 2011, p. 120). En este sentido, se encontró una significativa prevalencia de los comportamientos que favorecen un noviazgo violento: los celos, el control de las actividades que realiza la mujer, revisar sus objetos personales como el celular, en parejas con menos tiempo de relación.

En Colombia, la Universidad de Antioquia llevó a cabo un estudio de tipo mixto y de carácter multidisciplinario sobre violencia de género en el 2005, que posteriormente fue reeditado, de acuerdo a los autores, por la importancia y vigencia del tema (Fernández, 2013). El estudio señala que la violencia de género es una problemática estructural en el ámbito universitario debido a las inequidades que impone el mismo sistema educativo. Según los resultados obtenidos, tanto a administrativos, docentes como estudiantes, en el espacio universitario se reproducen relaciones excluyentes y discriminatorias que crean obstáculos para ejercer el poder, lo cual dificulta el cumplimiento de objetivos como la formación académica y profesional.

Además, dicha investigación revela que se presenta una invisibilización de las diversas expresiones de violencia de género en el contexto universitario. De acuerdo a los autores, dicha invisibilización en su mayoría es ocasionada por la oposición tradicionalmente establecida entre la vida académica y la violencia, bajo la idea que la formación intelectual constituye un factor protector ante las violencias. Sin embargo, los investigadores aclaran que se debe admitir que en las relaciones humanas y en cualquier ámbito que tengan lugar "existe la tendencia a la discriminación y a la exclusión de aquellos a los que se consideran inferiores y débiles" (Fernández et al., 2013, p. 9). En síntesis, la investigación develó un trato inequitativo entre géneros, dado que anteponía el modelo patriarcal, la masculinidad como centro y la prevalencia de la heterosexualidad.

Para ampliar la discusión, en el estudio realizado por Moreno, Sepúlveda y Restrepo (2013) se encontró una alta prevalencia de la violencia de género en la Facultad de Ciencias para la Salud de la Universidad de Caldas. La violencia se presenta tanto en los administrativos como en los docentes y estudiantes, cuyas manifestaciones más frecuentes fueron el abuso de autoridad, las burlas, los piropos y los gestos obscenos. En la misma región, pero con un estudio más amplio que abarcó cuatro universidades de Manizales, Cardona y otros (2015) muestran que la violencia contra las mujeres 
sigue siendo prevalente de forma significativa. En este artículo, las variables que se tuvieron en cuenta fueron: la calidad de relación entre los géneros en el programa, el sexismo hostil, el sexismo benevolente, la justificación de la violencia de género en el sistema patriarcal, justificación del sexismo y la violencia como reacción, la victimización de la pareja contra la mujer y los consejos de adultos sobre actitudes sexistas. Con cada una de éstas se aplicó una escala y se relacionaron. Los resultados obtenidos por Cardona y otros (2015) acerca de las creencias religiosas coinciden con hallazgos también de Castaño y otros (2010): "las personas en que la religiosidad es importante, las actitudes sexistas son más notables que aquellos que no son religiosos" (p. 217), pues hallaron diferencias significativas en el nivel de sexismo benévolo entre quienes profesan alguna religión y los que no, obteniéndose puntuaciones más altas en los primeros.

En Bogotá, de acuerdo a Orrego y Ariza (2014), resulta relevante la aprobación del Acuerdo 035 de 2012 que establece la política de equidad de género en la Universidad Nacional de Colombia, que, a su vez, ha permitido generar alianzas internacionales a través de proyectos como: Medidas para la Inclusión Social y Equidad en las Instituciones de Educación Superior en América Latina (MlSEAL) y Fortalecimiento de capacidades relacionadas con la equidad de género en la Educación Superior (FEGES) junto a otras universidades del país. FEGES no sólo fue una estrategia para el fortalecimiento de la equidad de género en los estudiantes, sino que focalizó muchos de sus esfuerzos en la capacitación de los docentes y administrativos. Esto, debido a la influencia que particularmente los profesores ejercen sobre los estudiantes con sus comentarios, sus modos de tratamiento y la forma en la que explican en sus clases, siendo ellos aún más ejemplo para los estudiantes en sus actitudes y percepciones, en este caso específico sobre la violencia de género. En el marco de este proyecto, en la Universidad Industrial de Santander, una investigación demostró que para las mujeres:

es más difícil el acceso a cargos de poder en la universidad [...], existe discriminación por sexo en cuanto al mayor valor que se otorga a la participación de los hombres en la academia y en la escritura científica (Rojas, Méndez \& Montero, 2013, p. 214).

\section{Estrategias comunicativas universitarias sobre violencia de género}

Para entrar en el contexto, es importante resaltar el trabajo realizado en la creación, y al mismo tiempo, en la crítica de las estrategias comunicativas gubernamentales, así como las de empresas privadas. Calandria y Marcos (2012) realizaron una investigación sobre las campañas de televisión frente a la violencia de género en España. Los resultados evidencian que la mayoría de población no considera que las campañas de televisión modifiquen su forma de pensar sobre el tema y eso es contrastado con el aumento del número de víctimas durante el 2010 de un 23\%, respecto al 2009. Algo similar sucedió con la investigación adelantada por Papí y Orbea (2011) quienes validaron un modelo para evaluar la eficacia de la publicidad comercial; ellos afirman que 
la publicidad institucional debe revisar la estrategia política y publicitaria de forma conjunta, dado que, cuando se toman por separado, sin estudios previos, los resultados no se ajustan a la realidad.

Específicamente en las universidades, se encontró un trabajo realizado en materia de prevención a través de la sensibilización en redes sociales. Un estudio hecho en la Fundación Universitaria Los Libertadores en Bogotá destacó las campañas publicitarias en las redes como herramientas de conciencia contra el acoso sexual hacia mujeres (Martínez \& Pérez, 2016). En el trabajo presentado por investigadores en publicidad y mercadeo, se revisaron las campañas nacionales e internacionales sobre violencia de género en las instituciones de educación superior y se propuso una en este claustro.

La mayoría de las campañas que Martínez y Pérez (2016) recopilaron se basaron en piezas gráficas distribuidas en las redes sociales y páginas institucionales, en las cuales, en su mayoría, conmemoraban el Día internacional de la violencia contra la mujer (25 de noviembre). Algunas presentaban concursos interactivos en redes sociales con hashtags "Crea tu eslogan y sube la foto" como en UNSAM en Argentina; otras presentaban muestras artísticas de pintura o teatro como en España, y otras, de más largo aliento, proponían jornadas de trabajo y sensibilización con conferencias y talleres. En Colombia, la campaña "Usa la razón" utilizó su propia página en Facebook y su contenido estaba basado en las canciones de reggaetón, cuyo lema era "que la música no degrade tu condición, Usa la Razón", de la Universidad Jorge Tadeo Lozano. Estas campañas descritas les permitieron a los investigadores crear una propia cuyo objetivo fue "sensibilizar y visibilizar el acoso sexual por parte de los hombres al que son expuestas las mujeres en la universidad" (Martínez \& Pérez, 2016) cuyo nombre fue Homo Cerdus.

También se encuentran otras campañas publicitarias impulsadas por colectivos no institucionalizados. Tal es el caso de la campaña "No es Normal", de la Universidad de los Andes (Tapia, 2017a) que, desde hace dos años, denuncia expresiones de violencia de género al interior del claustro universitario. El propósito de este colectivo conformado por profesores y estudiantes es visibilizar muchas de las actitudes y acciones cotidianas que son violencia de género a través de frases o textos muy cortos publicados en un formato gráfico, los cuales fueron compartidos en carteleras de las Facultades y en las redes sociales. En unión con PACA, Pares de Acompañamiento Contra el Acoso, "No es Normal" ha captado la atención de los estudiantes y, haciendo uso de las redes y el diseño, han logrado traducir los discursos feministas en acciones colectivas de impacto. Tanto es así que, en el último año, universidades como el Externado de Colombia y la Pontificia Universidad Javeriana se han sumado a la campaña en sus respectivas instituciones.

Con más antigüedad, también se encuentra el Colectivo Blanca Villamil de la Universidad Nacional con su campaña "En la Nacho no más machos" (Tapia, 2017a), que surge ante el acoso sexual en el campus. A través de diversos testimonios se evidenciaron las continuas expresiones de superioridad masculina, el acoso callejero en los pasillos del campus, los comentarios desobligantes por parte de profesores hombres, entre otros abusos de poder. 
Asimismo, en la Universidad Nacional, la Escuela de Estudios de Género hizo una alianza con Bienestar Universitario recientemente y juntos realizaron una campaña denominada "Parece trivial, pero no lo es" (Bienestar UN, 2016) que consistió en cuatro piezas gráficas, tipo caricatura, en las cuales se evidenciaban casos recurrentes de violencia contra las mujeres con frases muy comunes que ejercen violencia simbólica (- ¡Buenos días! Vengo a radicar estos documentos. - ¡Están como bien de funcionarias en esta dependencia!, deberías venir aquí más seguido...).

Además, la campaña "Parece trivial pero no lo es" circuló en internet a través de redes sociales y correos electrónicos instituciones y recorrió el campus con las piezas impresas tanto en las aulas, los sitios comunes de los estudiantes, así como las oficinas de profesores, administrativos y demás empleados de la universidad, articulando de esta forma a todos los miembros de la comunidad universitaria. Esta campaña se dio a conocer en el mes de noviembre y coincidió así con el Día internacional de la violencia contra las mujeres, e hizo parte de debates y reflexiones en la jornada "Hacer y deshacer la ideología de género", organizada por la Escuela de Estudios de Género de esa casa de estudios (Bienestar UN, 2016).

La anterior campaña hace parte de un proyecto de convivencia y cultura universitaria que contó además con un estudio que exploró a profundidad la violencia sexual hacia las mujeres estudiantes de la Universidad Nacional de Colombia. Esta información se recopiló en el informe "Rompiendo el silencio" (Rozo, 2016), en el cual, entre otros resultados de la encuesta general, evidenciaron que la forma más común de violencia sexual se da a través de "piropos o comentarios sexuales, este tipo de violencia tiene la característica de ser la más naturalizada, lo que tiene que ver con que sea menos denunciada, aunque genere rabia, impotencia e incomodidad" (Rozo, 2016, p. 26). En la Universidad Javeriana, sede Bogotá, también funciona el Colectivo Polifonía (Tapia, 2017a), un colectivo de género que piensa el contexto universitario en clave de género.

En la misma línea trabaja "Rosario Sin Bragas", un colectivo estudiantil feminista de la Universidad del Rosario en Bogotá (Tapia, 2017a) que desde el 2012, ante la ausencia de espacios institucionales para discutir asuntos de género, creó este espacio (Tapia, 2017b). Los objetivos fundamentales del colectivo tienen que ver con la reflexión de las desigualdades y encontrar estrategias y alternativas para visibilizar la violencia al interior de las universidades. Para esto, realizan actividades artísticas en las fechas clave que recuerdan a las mujeres y han avanzado hacia la creación de una escuela que ofrece talleres y cursos sobre temas feministas y de género. Este colectivo creó una plataforma permanente denominado "Alerta Violeta" que sirve para realizar denuncias, contar testimonios y así visibilizar la violencia de género dándole voz a las mujeres que han sido víctimas en el contexto universitario.

Cabe señalar que actualmente en el ámbito latinoamericano se está viviendo un proceso de visibilización y sensibilización de la violencia de género apoyado en gran parte por los grupos universitarios y de mujeres profesionales. Campañas como \#MiPrimerAcoso (Sánchez, Milizaliturri \& Casanueva, 2016) en redes sociales y manifestaciones como el \#NiUnaMenos 
(3 de junio de 2015) o el \#MiércolesNegro (19 de octubre de 2016) y el Día de la Mujer el 8 de marzo de 2017, que dio lugar a un Paro Internacional de mujeres ("el \#8M"), están utilizando estrategias comunicativas en redes sociales que se han traducido en miles de mujeres manifestándose en las calles y siendo noticia en los medios de comunicación tradicionales. Ambas movilizaciones, las virtuales y las del espacio público físico, hacen un llamado a la comprensión de una problemática que, sin duda, no afecta solamente a las mujeres, pues la violencia hacia a ellas genera un impacto en la vida social de todos los individuos.

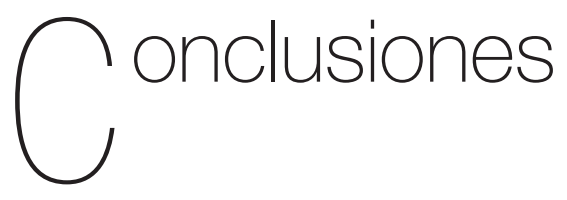

El problema de la violencia de género en las universidades es una tendencia en desarrollo dentro de la investigación en ciencias sociales. Se ha abordado desde diversas disciplinas: psicología, educación, sociología antropología, historia y desde las ciencias de la salud. Dichas investigaciones han permitido que se alerte a las instituciones de educación superior y, en general, a toda la comunidad académica, para construir estrategias hacia la resolución de esta problemática. Entre los aspectos para enfrentar la violencia de género se encuentran la prevención, la atención y sanción de los casos; y, para esto, una de las mejores herramientas es la comunicación, porque permite informar y al mismo tiempo explicar el problema para prevenirlo.

Las investigaciones aquí mencionadas evidencian la importancia y la urgencia de prestar atención a la violencia de género en las universidades, considerando sus distintas manifestaciones: violencia de tipo sexual, física, psicológica, en relaciones de pareja, el noviazgo entre estudiantes, las relaciones laborales entre la comunidad académica, entre otras. Se concluye que la universidad no está exenta, como se creía antes, de presentar situaciones de violencia; de hecho, es un espacio que puede llegar a propiciar violencia por actos de omisión.

La universidad puede ser un territorio peligroso para las mujeres por la exposición que tienen a la violencia (Flack et al., 2008), pero, también es cierto que se puede configurar en un espacio donde se agencie la igualdad de género (Ingrassia, 2017) y se propicien prácticas dialógicas que promuevan la igualdad de los géneros, y, al mismo tiempo, mujeres empoderadas: "Se considera imperiosa la mayor inclusión de teorías y prácticas feministas, de conceptos relacionados con la igualdad de oportunidades y de autorías femeninas en las bibliografías" (Ingrassia, 2017, p. 67). Su planteamiento se basa en la idea de la educación como agente de acción cultural cuyo objetivo debe ser la concientización política y social. En esa medida, la universidad debe desnaturalizar las creencias y conductas aprendidas que propician la violencia y así transmitir esos saberes a la sociedad a través de los profesionales que más tarde harán parte de ella.

Ante esta realidad, se hace necesario que las universidades asuman la responsabilidad de la socialización preventiva (Valls \& Prados-Gallardo, 2014). Se debe impulsar a las universidades a "asumir el liderazgo en la tarea de escrutar rigurosamente las agresiones, establecer prioridades, y refinar los estándares del 
consentimiento sexual entre personas adultas" (Rubio, 2015, p. 1); dado que, como se evidenció, las instituciones universitarias pueden llegar a dificultar que las mujeres víctimas denuncien a sus agresores por su naturaleza jerárquica y el dominio de los hombres en las estructuras de poder (Valls, 2008). Se habla entonces del deber de las instituciones de educación superior (Ley 1257 de 2008) para crear entornos libres de discriminación y acoso donde sea posible una convivencia armónica y donde los conflictos sean gestionados sin violencia.

En cuanto a las estrategias comunicativas que diseñan e implementan las universidades para la prevención de la violencia de género, se encontró que son muy escasas las registradas en artículos de investigación. Es decir, hay un gran avance en materia de investigación, como se vio anteriormente, pero se presenta escaso registro sobre el enorme trabajo que seguramente realizan las secciones de Bienestar Universitario y los colectivos internos de las universidades en la visibilización de la violencia de género en los claustros universitarios. Una de las razones que se plantean es que no están articuladas con grupos de investigación, ni basadas en los resultados que arrojan estudios y que, además, se realizan de forma esporádica, sin un hilo conductor más que la conmemoración de fechas como el 8 de marzo, o a raíz de puntos de inflexión como la agresión a alguien en particular.

Dentro de los resultados del presente trabajo se puede evidenciar el hallazgo de estrategias comunicativas que revelan la violencia contra las mujeres que, en su mayoría, han sido movimientos de abajo hacia arriba. Es decir, la problemática se ha visibilizado en las aulas, los pasillos, las plazoletas, las redes (Vázquez, 2015) y poco a poco ha llegado a impactar a la comunidad universitaria; es así como el tema ha logrado posicionarse como un eje fundamental de discusión en los claustros. Sin embargo, a pesar de esos esfuerzos, dichas estrategias aún no se traducen en políticas al interior de las instituciones educativas que atiendan y, sobretodo, prevengan de forma contundente este tipo de violencia.

Según lo revela un especial realizado por el portal periodístico "Distintas Latitudes" (Meléndez, Ilizaliturri \& Pagola, 2016), de 63 universidades de América Latina escrutadas, solo 14 tienen un protocolo o una ruta de atención frente a la violencia de género. De hecho, periódicos alternativos colombianos como "Vice" han llamado la atención cuestionando esta realidad con artículos como: "¿Y por qué los rectores no están hablando de abuso sexual en las universidades?" (Tapia, 2017a) y con notas testimoniales sobre la violencia (Tapia, 2017b) que han sufrido estudiantes de los claustros de educación superior. De esta manera, se evidencia que enfrentar la problemática de la violencia de género en las universidades supone convocar a los miembros de la comunidad a una transformación de sus imaginarios culturales (Bosh-Fiol \& Ferrer-Pérez, 2012), los discursos (Matynowsky, 2015) y, en general, de conductas (Larena \& Molina, 2010) que promueven en la población académica espacios libres de violencia contra las mujeres.

Se discute así la articulación que se debe establecer entre la investigación, la comunicación y las autoridades universitarias para combatir la violencia de 
género. La articulación de estos tres estamentos y de la mano del activismo deben desencadenar en la construcción de políticas universitarias que, a su vez, generen protocolos de prevención, atención y sanción (Mérida, Salazar \& Agudo, 2015), pasos que ya han empezado a dar a algunas universidades latinoamericanas. Cabe decir que una de las universidades más prestigiosas del mundo ya lo hizo (Harvard, 2014) intentando, de esta forma, enfrentar la idea del desprestigio institucional.

Las universidades en Argentina han dado un paso más allá con la creación de la Red Interuniversitaria (Vázquez, 2015), con la cual se puede generar un mayor respaldo para que todas las instituciones garanticen las rutas de atención que les permitan encontrar alternativas a la violencia y, en el mejor de los casos, lograr prevenirla antes de que suceda a través de la información a tiempo. Esto último es lo que están empezando a lograr a través de las oficinas de apoyo en las universidades, es decir, lugares donde se les preste atención y se conduzcan los procesos de acompañamiento a quienes son víctimas de abusos en los claustros. Estos han sido los resultados en las universidades norteamericanas, donde han avanzado en la lucha contra la violencia sexual que se vive en los campus (Jordan, 2011; Palaudi, 2016; Schewe et al., 2011). Es el trabajo en equipo el que permite la integración de los esfuerzos de los centros de atención con los avances académicos y los centros de investigación para prestar óptimos servicios preventivos y generar planes y proyectos articulados para obtener mejores resultados.

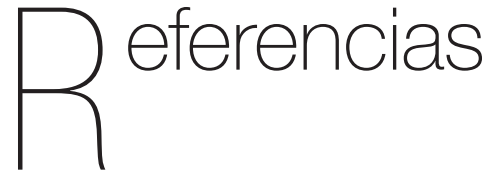

Acosta, M. (2015). Comportamiento de la Violencia de Pareja: Colombia 2015. En Revista Forensis. Grupo Centro de Referencia Nacional sobre la Violencia. Instituto Nacional de Medicina Legal y Ciencias Forenses (INML). Bogotá.

Aguilar, C., Alonso, M. J., Melgar, P. \& Molina, S. (2009). Violencia de género en el ámbito universitario. Medidas de superación. Revista Interuniversitaria de Pedagogía social, 16(1), 85-94.

Amnistía Internacional. (2017). Las mujeres sufren discriminación, ya sea institucionalizada por ley o en la práctica, incluso en países con leyes que garantizan la igualdad. Recuperado de https://www. es.amnesty.org/en-que-estamos/temas/ mujeres/.

Barbosa, P. (2015). La perspectiva de género en las instituciones de educación superior públicas en México frente a los problemas de desigualdad y violencia en razón de género. En O. Rojas \& L. Ibarra. (Comps.) Estado, Derecho y Desigualdad (pp. 127153). Michoacán, México: Universidad Michoacana de San Nicolás de Hidalgo.

Barrancos, D., Torricella, P. \& Vázquez, V. (2015). Se lanza en la UNSAM la Red interuniversitaria por la igualdad de género y contra las violencias. Programa contra la violencia de género, PCVG. Universidad Nacional de San Martín. Recuperado de http://www.unsam.edu.ar/pcvg/pdf/ Revista2015.pdf 
Barredo, D, Cevallos, M. \& Zurbano, B. (2014). El concepto de violencia de género en el imaginario latinoamericano: estudio sobre los universitarios quiteños. En J. Suárez, R. Lacalle, \& J.M. Pérez., (Eds.). Trabajo presentado en II International Conference Gender and Communication. (pp. 462472). Madrid: Dykinson S. L.

Berenguera, A. Fernández, M. J., Pons, M. Pujol, E., Rodríguez, D. \& Saura, S. (2014). Escuchar, observar y comprender. Recuperando la narrativa en las Ciencias de la Salud. Aportaciones de la investigación cualitativa. Barcelona: Institut Universitari d'Investigació en Atenció Primera Jordi Gol.

Bernal, M. (21 de mayo 2016). El silencio, cómplice del acoso sexual en las universidades del país. El Tiempo. Recuperado de http://www.eltiempo. com/politica/justicia/cifras-sobreacoso-sexual-en-las-universidades-decolombia/16598964

Bienestar UN. (25 de noviembre 2016). iPongamos fin a las violencias contra las mujeres! Boletín UN. Universidad Nacional de Colombia. Recuperado de http:// bienestar.bogota.unal.edu.co/ver_noticia. php?id_noticia=483.

Bosh-Fiol, E. (2009). El acoso sexual en el ámbito universitario. Elementos para mejorar la implementación de medidas de prevención, detección e intervención. Universidad de las Islas Baleares. Grupo de investigación en estudios de género. Recuperado de http://www. inmujer.gob.es/areasTematicas/estudios/ estudioslinea2014/docs/El_acoso_sexual_ ambito_universitario.pdf.

Bosh-Fiol, E. \& Ferrer-Pérez, V. (2012). Nuevo mapa de los mitos sobre la violencia de género en el siglo XXI. Psicothema, 24(4), 548-554.

Bourdieu, P. (2000). La dominación masculina. Barcelona: Anagrama.

Buquet, A. (2011). Transversersalización de la perspectiva de género en la Educación superior. Problemas conceptuales y prácticos. Perfiles Educativos, 33(1), 211-225.

Buquet, A., Cooper, J., Mingo, A. \& Moreno, $\mathrm{H}$. (2013). Intrusas en la Universidad. México D.F.: Universidad Nacional Autónoma de México. Recuperado de www.pueg.unam. $\mathrm{mx}$.

Calandria, E. \& Marcos, M. (2012). Campañas en televisión contra la violencia de género del ministerio de sanidad, política social e igualdad (2008-2011). Análisis de contenidos previo al estudio de recepción. Revista de Comunicación Vivat Academia, 121(1), 17-30.

Cardona, J., Casa, L., Cañón, S., Castaño, J., Godoy, A., Henao, D., \& Valencia, L. (2015). Sexismo y concepciones de la violencia de género contra las mujeres en cuatro universidades de la ciudad de Manizales (Colombia). Archivos de Medicina, 15(2), 200-219.

Carvajal, Z. \& Delvó, P. (2010). Universidad Nacional: Reacciones y efectos del hostigamiento sexual en la población estudiantil en el 2008. Revista de Ciencias Sociales Universidad de Costa Rica, 4(1), 59-74.

Castaño-Castrillón Castaño-Castrillón, J., González, E., Guzmán, J., Montoya, J., Murillo, J., Páez, M., Parra, L., Salazar, T. \& Velásquez, Y. (2010). Acoso sexual en la 
comunidad estudiantil de la Universidad de Manizales (Colombia) 2008. Estudio de corte transversal. Revista Colombiana de Obstetricia y Ginecología, 61(1), 18-27.

Castro, R. \& Vázquez, V. (2008). La Universidad como espacio de reproducción de la violencia de género. Un estudio de caso en la Universidad Autónoma de Chapingo, México. Estudios Sociológicos, 26(78), 587-616.

Consejería Presidencial para la Equidad de la Mujer (2015). Avances de la tolerancia social e institucional de las violencias contra las mujeres en Colombia. Boletín No. 19. Recuperado de http://www.equidadmujer. gov.co/oag/Documents/oag_boletin-19marzo2015.pdf.

Chávez, J. (2015). Percepción de la igualdad de género en jóvenes universitarios. Trabajo Social UNAM, 10(4), 75-90.

Chávez, J. \& Rodríguez, A. (2015). Percepción de la violencia de género en las y los jóvenes universitarios. Trabajo social Sin Fronteras, 7(14), 63-74.

Domínguez, A., Fernández, N. \& Fuentes, S. (2013). ¿Violencia de género en estudiantes de la Universidad Nacional de Córdoba? Recuperado de http://conferencias. unc.edu.ar/index.php/ponencias/ ponencias2013/paper/view/1754/604.

Echeburúa, E., Amor, P., Sarasua, B., Zubizarreta, I., \& Holgado-Tello, F. (2016). Inventario de pensamientos distorsionados sobre la mujer y el uso de la violenciarevisado (IPDMUV-R): propiedades psicométricas. Anales de Psicología, 32(3), 837-846.
Fernández, D. (2008). Gramáticas de la publicidad sobre violencia: la ausencia del empoderamiento tras el ojo morado y la sonrisa serena. Feminismo/s, 11(1), 15-39.

Fernández, J. (2013). Análisis y evolución de las campañas publicitarias promovidas por el gobierno de España tras la aprobación del Plan Nacional de Sensibilización y Prevención de la Violencia de Género. Pensar la Publicidad, 7(2), 409-424.

Fernández, S., Hernández, G. \& Paniagua, R. (2013). Violencia de género en la Universidad de Antioquia. $2^{\mathrm{a}}$ Edición. Medellín: Lealón.

Ferrer, V., Bosh-Fiol, E., Ramis, C., Torres, G., \& Navarro, C. (2006). La violencia contra las mujeres en la pareja y actitudes en estudiantes universitarios/as. Psicothema, 18(3), 359-366

Flack, W., Caron, M., Leinen, S., Breitenbach, K., Barber, A. M., Brown, E. N., Gilbert, C., Harchack, T., Hendricks, M., Rector, C., Schatten, H., \& Stein, H. (2008). "The red zone": Temporal risk factors for unwanted sex among college students. Journal of Interpersonal Violence, 32, 1177-1196.

Harvard University (2014). Interim Sexual Harassment Policy and Procedures [archive PDF] Cambridge, Massachusetts. Recuperado de http://hls.harvard.edu/ content/uploads/2014/09/hls-titleixinterimpolicy1.pdf.

Hayes-Smith, R. (2010). Student Perceptions of sexual assault resources and prevalence of rape myth attitudes. Feminist Criminology, 5(4), 335-354. 
Hernández, R., Fernández, C., \& Baptista, P. (2010). Metodología de la investigación. Madrid: McGraw-Hill.

Hoxmeier J. C., Flay B. R., \& Acock, A. C. (2016). Control, norms, and attitudes: Differences between students who do and do not intervene as bystanders to sexual assault. Journal of Interpersonal Violence. Advance Online Publication, 33(15), 23792401. doi:10.1177/0886260515625503.

Ingrassia, P. (2017). La universidad como agente de igualdad de género. RevIISE, 9(9), 63-69. Recuperado de http://www. ojs.unsj.edu.ar/index.php/reviise/article/ view/135/pdf.

Jordan, C. (2011). The University of Kentucky Center for research on violence against women: Science inspired by women's stories. Violence Against Women, 17(9), 1137-1158.

Larena, R. \& Molina, S. (2010). Violencia de géneroenlasuniversidades: Investigaciones y medidas para prevenirla. Trabajo social global. Revista de investigaciones en intervención social, 1(2), 202-219.

Ley 1257 de 2008: Código Penal. Congreso. Recuperado de http://www.sdmujer.gov. co/images/pdf/ley1257.pdf

Ley 1542 de 2012, por la cual se reforma el artículo 74 de la ley 906 de 2004, código de procedimiento penal. Diario Oficial 48482 de la República de Colombia, Bogotá, Colombia, 5 de julio de 2012. Recuperado de http://www.alcaldiabogota.gov.co/ sisjur/normas/Norma1.jsp?i=48239.

Ley 1761 de 2015, Rosa Elvira Cely: Código Penal. Congreso. Recuperado de http:// www.alcaldiabogota.gov.co/sisjur/normas/ Norma1.jsp?i=62278.

Lorenzo, M. \& Salazar, A. (2011). La violencia de género en el noviazgo. Un estudio descriptivo a partir de estudiantes universitarias. (Trabajo de grado). Recuperado de http://biblioteca2.ucab. edu.ve/anexos/biblioteca/marc/texto/ AAS3029.pdf

Martínez, H. \& Pérez, J. (2016). Las campañas publicitarias en social media como herramientas de concienciación contra el acoso sexual hacia las mujeres. (Trabajo de grado). Recuperado de http://repository.libertadores.edu.co/ handle/11371/633?mode=full

Matynowsky, E. (2015). Locos, psicópatas, anormales... O de las estrategias discursivas para invisibilizar el carácter estructural de la violencia de género. Temas y Debates, 30(19), 161-174.

Meléndez, J., llizaliturri, A. Pérez, D., \& Pagola, F. (27 de noviembre de 2016). Violencia sexual en las universidades de América Latina: Omisiones, obstáculos y opacidad. Revista Distintas Latitudes. Recuperado de http://distintaslatitudes.net/violenciasexual-universidades-america-latina.

Mérida, R., Salazar, O. \& Agudo, M. (2015). Protocolo para la prevención y protección frente al acoso sexual y por razón de sexo en la Universidad de Córdoba. Recuperado dehttps://www.uco.es/igualdad/protocolo/ documentos/protocolo-prevencion-v2.pdf.

Miracco, M. \& Lanzillotti, A. (2016, noviembre). Creencias y conductas vinculadas con la violencia en el noviazgo. Un estudio sobre estudiantes universitarios de la carrera de psicología. Trabajo presentado en el VIII 
Congreso Internacional de Investigación y práctica profesional de Psicología. Buenos Aires: UBA.

Mohler-Kuo, M., Dowdall, G. W., Koss, M. P., \& Wechsler, H. (2004). Correlates of rape while intoxicated in a national sample of college women. Journal of Studies on Alcohol, 65, 37-45.

Moral, J. \& Ortega, M. (2008). Diferencias de género en significados, actitudes y conductas asociados a la sexualidad en estudiantes universitarios. Estudios sobre las culturas Contemporáneas, 28(14), 97-119.

Moreno, C. Sepúlveda, L. \& Restrepo, L. (2013). Prevalencia de la violencia y discriminación contra la mujer en la facultad de ciencias para la salud, Universidad de Caldas, Colombia, 2010-2011. Revista colombiana de obstetricia y ginecología, 64(1), 12-20.

Naciones Unidas, Asamblea General (1999, 6 de octubre). Protocolo facultativo de la convención sobre la eliminación de todas las formas de discriminación contra la mujer. Recuperado de http://www. ohchr.org/SP/Professionallnterest/Pages/ OPCEDAW.aspx.

Naciones Unidas, Asamblea General (2015, 12 de agosto). Transformar nuestro mundo: la Agenda 2030 para el desarrollo sostenible. Recuperado de http://www.socialwatch. org/sites/default/files/Agenda-2030-esp. pdf.

Naciones Unidas. (2013, 1 de junio). Objetivos de desarrollo del milenio. Informe de 2013. Nueva York. Recuperado de http:// www.un.org/es/millenniumgoals/pdf/mdgreport-2013-spanish.pdf
Oliver, E. \& Valls, R. (2004). Violencia de Género. Investigaciones sobre quiénes, por qué y cómo superarla. Barcelona: Hypatia Editorial.

Ordorika, I. (2015). Equidad de género en la Educación Superior. Revista de la Educación Superior, 44(2), 7-17.

Organización Internacional del Trabajo (OIT) (2005). Glosario de términos básicos para comprender y analizar el mercado de trabajo desde la perspectiva de la equidad de género. Recuperado el 17 de enero de 2017 de http://portal. oit.or.cr/index.php?option=com staticxt\&staticfile=genero/ glosarioarreglado181.08.04.pdf.

Organización Mundial de la Salud (OMS) (2014). Violence against women. Fact sheet N239. Recuperado de http://www.who.int/ mediacentre/factsheets/fs239/en/.

Orrego, L. \& Ariza, G. (2013). La violencia de género en las universidades colombianas. Teoría \& Sociedades, 21(2), 135-156.

Palaudi, M. (2016). Campus action against sexual assault: Needs, policies, procedures and training programs. California: Abc-clio.

Papí, N\& Orbea, J. (2011). La eficacia publicitaria de las campañas sobre la igualdad de género: análisis de la codificación y del plan de medios. Zer, 16(30), 137-266.

Rodríguez, Y., Lameiras, M, Carrera, M., \& Alonso, P. (2014). Análisis de la violencia de género en estudiantes de las universidades gallegas. III Jornada de Innovación en género. Docencia e Investigación. Universidad de Vigo, pp. 287-302. 
Rojas, M., Méndez, R. \& Montero, L. (2013). Satisfacción laboral y relaciones de género en la Universidad Industrial de Santander. Revista Virtual Universidad Católica del Norte, 40(1), 204-2015.

Romero-Sánchez, M, Megías, J., \& Krahé, B. (2012b). The role of Alcohol and victim sexual interest in Spanish students' perceptions of sexual assault. Journal of Interpersonal Violence, 27(11), 2230-2258.

Romero-Sánchez, M., López, J., CarreteroDios, H. \& Rincón, L. (2012a). Versión colombiana de la escala Acceptance of Modern Myths about Sexual Aggression Scale: primeros análisis psicométricos. Revista Latinoamericana de Psicología, 45(1), 121-134.

Rozo, L. (2016). Rompiendo el silencio. Análisis de encuesta sobre violencia sexual a estudiantes mujeres. Bogotá, D.C.: Dirección de Bienestar Universidad Nacional de Colombia.

Rubio, M. (11 de diciembre de 2015). Universidad y violencia sexual. El Diario Bogotano Recuperado de http://www.eldiariobogotano.com/ universidad-y-violencia-sexual/.

Sánchez, M., llizaliturri, A. \& Casanueva, J. (24 de mayo 2016). \#MiPrimerAcoso: la etiqueta que destapó la cloaca de las agresiones sexuales. En Distintas Latitudes, Dossier, género. Recuperado de http://distintaslatitudes.net/miprimeracosola-etiqueta-que-destapo-la-cloaca-de-lasagresiones-sexuales.

Scott, J. (1996). El género: Una categoría útil para el análisis histórico. En M. Lamas (Comps.), El género: La construcción cultural de la diferencia sexual. (pp. 265302) México D.F.: PUEG.

Schewe, P., Bell, C., Bennett, L., Goldstein, P., Gordon, R., Mattaini, M., O’Brien, P., Riger, S., Healther, R., Rosenbaum, D., Schukc, A., Simmons B., \& Ullman, S. (2011). University of Illinois at Chicago's Interdisciplinary Center for Research on Violence: Changing Systems to Prevent Violence in Chicago and Beyond. Violence Against Women, 17(9), 1176-1193.

Strauss, M. (2004). Prevalence of violence against dating partners by male and female University Students worldwide. Violence against Women, 10(7), 790-811.

Tapia, T. (24 de enero de 2017b). Abuso sexual en las universidades de Colombia: un enemigo silencioso. Recuperado de https://www.vice.com/es_co/article/acososexual-en-las-universidades-de-colombiaun-enemigo-silencioso.

Tapia, T. (5 de febrero de 2017a). ¿Y por qué los rectores no están hablando de abuso sexual en las universidades? Recuperado de https://www.vice.com/es_co/article/ypor-que-los-rectores-no-estan-hablandodel-abuso-sexual-en-las-universidades.

Valls, R. (2008). Violencia de género en las universidades españolas. Ministerio de Igualdad: Universidad de Barcelona.

Valls, R. \& Prados-Gallardo, M. (2014). Socialización preventiva en la violencia de género. En R. M. Radl Philipp \& M.C. Fonseca-Silva (Eds.), Violencia contra las mujeres: perspectivas transculturales (pp. 151-169). Universidad Santiago de Compostela. 
Valls, R., Oliver, E., Sánchez, M., Ruiz, L., \& Melgar, P. (2007). ¿Violencia de género también en las universidades? Investigaciones al respecto. Revista de Investigación Educativa, 25(1), 219-231.

Valls, R., Puigvert, L., Melgar, P., \& García-Yeste, C. (2016). Breaking the silence at Spanish universities: Findings from the first study of violence against women campuses in Spain. Violence Against Women, 22(13), 1519-1539.
Vásquez, V. (2015). Ya somos una Red Interuniversitaria por la lgualdad de Género y contra las violencias. UNSAM en la Red. Recuperado de http://www.unsam.edu.ar/ pcvg/pdf/Revista2015.pdf.

Vasquez, V., Palumbo, M., \& Fernández, C. (2016). ¿Cómo prevenir, sancionar y erradicar la violencia de género en las universidades? Revista Ciencias Sociales, 92, 106-114. 